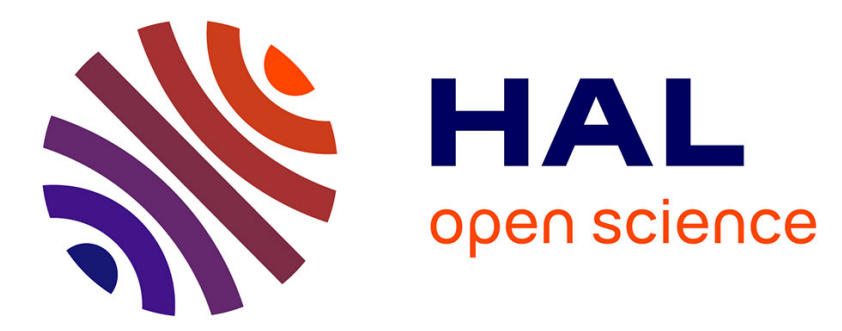

\title{
La participation au service du néolibéralisme? Les usagers dans les services d'eau en Afrique subsaharienne
}

Sylvy Jaglin

\section{To cite this version:}

Sylvy Jaglin. La participation au service du néolibéralisme? Les usagers dans les services d'eau en Afrique subsaharienne. BACQUÉ Marie-Hélène, REY Henri, SYNTOMER Yves. Gestion de proximité et démocratie participative : une perspective comparative, La Découverte, pp.271-291, 2005, Recherches. halshs-00182510

\section{HAL Id: halshs-00182510 \\ https://shs.hal.science/halshs-00182510}

Submitted on 26 Oct 2007

HAL is a multi-disciplinary open access archive for the deposit and dissemination of scientific research documents, whether they are published or not. The documents may come from teaching and research institutions in France or abroad, or from public or private research centers.
L'archive ouverte pluridisciplinaire HAL, est destinée au dépôt et à la diffusion de documents scientifiques de niveau recherche, publiés ou non, émanant des établissements d'enseignement et de recherche français ou étrangers, des laboratoires publics ou privés. 
La participation au service du néolibéralisme ? Les usagers dans les services d'eau en Afrique subsaharienne

Sylvy Jaglin

In BACQUÉ Marie-Hélène, REY Henri, SYNTOMER Yves (dirs.), 2005 Gestion de proximité et démocratie participative : une perspective comparative Paris, La Découverte, pp. 271-291 ISBN : 2-7071-4306-5 


\title{
LA PARTICIPATION AU SERVICE DU NEOLIBERALISME ? LES USAGERS DANS LES SERVICES D'EAU EN AFRIQUE SUBSAHARIENNE
}

\author{
Sylvy JAGLIN \\ Latts (UMR 8134) \\ ENPC, 6 et 8 avenue Blaise Pascal - Cité Descartes \\ F 77455 Marne-La-Vallée cedex 2 \\ e-mail : jaglin@enpc.fr
}

Nous nous proposons ici d'analyser les politiques de participation des usagers dans les services d'eau urbains d'Afrique subsaharienne, dont la faillite gestionnaire est patente. Hors de l'Afrique du Sud et de la Namibie, les taux de desserte demeurent faibles, inférieurs à $41 \%$ en moyenne, à peine supérieurs à $55 \%$ dans les grandes villes (plus de 1 million d'habitants), de $27 \%$ seulement pour les pauvres (Demographic and Health Surveys). Dépourvus de service ou confrontés à ses défaillances, les citadins sont, dans leur majorité, livrés aux marchés informels de l'eau et à des solutions individuelles.

Pour remédier à cette situation, des réformes ont été engagées dans les années 19801990. D'abord, en « rapprochant» les gestionnaires de réseau des usagers, la décentralisation est censée accroître la responsabilisation locale dans les systèmes de fourniture d'une part, améliorer l'efficacité des politiques tarifaires et d'investissement d'autre part. Les réformes africaines ont cependant démontré la difficulté à concrétiser une relation probante entre responsabilisation des politiciens et techniciens locaux d'une part, efficacité de leur action d'autre part, donc à traduire en termes matériels les progrès de la démocratie.

Ensuite, la marchandisation des services d'eau, attestée par les mutations des normes et règles de leur gestion (tarification au coût « complet », principe d'usager-payeur) et par le remaniement des dispositifs d'organisation (corporatisation, partenariats public/privé, libéralisation), est supposée accroître les performances économiques des opérateurs. Cependant, elle pose des problèmes d'efficacité redistributive, alors même qu'est réaffirmé le rôle stratégique des services en réseau dans la réduction de la pauvreté et des inégalités socio-spatiales (Banque mondiale, 1994).

Pour dépasser ces contradictions et la crise de confiance qui s'ensuit entre les usagers citoyens et leurs services publics, la participation est aujourd'hui présentée comme un élément indispensable à la recomposition de l'action collective en ville. "Chaînon 
manquant » des réformes antérieures, elle permettrait à la fois de renforcer l'efficacité des investissements et de la gestion d'une part, de promouvoir la démocratisation et l'équité des choix collectifs par l'empowerment des populations vulnérables de l'autre. Pourtant, si des preuves tangibles d'une contribution de la participation à l'amélioration des conditions de vie matérielles des pauvres en ville ont été apportées ici et là, son efficacité comme stratégie de changement social demeure en question. En d'autres termes, qu'elle favorise des progrès de la desserte ne suffit pas à démontrer sa capacité à réguler de nouveaux systèmes de solidarité aptes à conjurer les effets délétères de la marchandisation des réseaux. En s'appuyant sur des travaux consacrés à l'action publique et collective dans les services d'eau urbains en Afrique subsaharienne (Jaglin, 2003), cet article propose d'interroger les raisons qui suscitent aujourd'hui le déploiement d'une intense ingénierie participative ainsi que les conditions et modalités de sa mise en œuvre.

D’autres études, consacrées aux pratiques des communautés citadines, mettent l'accent sur le dynamisme des populations et leur capacité à « réveiller» les routines politicoadministratives. Certaines interrogent la nature des relations construites avec les autorités et soulignent la nécessité de dépasser une approche binaire, entre modes de coopération et formes de confrontation, pour saisir la diversité de leurs combinaisons à l'échelle locale (Oldfield, 2003). Privilégiant une réflexion sur la gestion urbaine, notre propos s'inscrit davantage dans une critique de la participation comme catégorie de l'action publique. Il rejoint ainsi les conclusions d'auteurs qui dénoncent une «nouvelle tyrannie » de la pensée et de la pratique du développement (Cooke, Kothari, 2001), soulignent les limites des dispositifs « communautaires » dans la gestion des biens et services collectifs (Cleaver, 2001 ; Manor, 2002) ainsi que leur ambivalence dans les processus de marchandisation (Page, 2003). À l'encontre des discours dominants sur la question, alimentés par une vision idéalisée de la gestion participative, notre hypothèse est en effet que les expériences africaines actuelles ont davantage pour souci d'intégrer les pauvres, ou une fraction d'entre eux, dans le marché que de promouvoir une gouvernance urbaine démocratique, assurant la représentation d'intérêts pluralistes et souvent antagoniques.

\section{LES FONDEMENTS RENOUVELES D'UNE PARTICIPATION PROTEIFORME}

Dans la littérature scientifique comme dans les documents politiques, les réformes des services publics et celles qui concernent la démocratie de proximité sont le plus souvent traitées séparément. Dans les pays en développement, les premières ont ainsi suscité la production d'un volumineux corpus centré sur l'introduction des principes du marché et celle du secteur privé dans les nouveaux dispositifs organisationnels. Les secondes sont traitées dans une abondante littérature sur la « société civile " ${ }^{1}$ et les mouvements sociaux. Notre posture résulte au contraire de la conviction qu'une réforme efficace des services doit s'appuyer sur des institutions, formelles et informelles, largement ouvertes à la participation de tous les usagers mais aussi que, à l'inverse, l'ampleur et l'efficacité

\footnotetext{
${ }^{1}$ Cette notion de société civile, éminemment polysémique, est à prendre avec précaution : les africanistes, politologues en particulier, demeurent sceptiques sur la possibilité de la transposer dans des cadres politiques marqués par le patrimonialisme, l'indifférenciation entre sphère privée et publique (Pirotte, Poncelet, 2002).
} 
de celle-ci sont étroitement dépendantes des caractéristiques des services publics (Goetz, Gaventa, 2001, p. 3).

L'appropriation du répertoire participatif par l'approche managériale ${ }^{2}$ en vigueur dans la réforme des services n'est toutefois pas sans poser problème. Elle induit notamment des glissements de sens et des simplifications sources de nombreuses confusions et, plus généralement, suscite une dépolitisation du débat sur la place et le rôle des usagers.

\section{A. La participation des citadins au développement urbain : une histoire ancienne}

L'idée d'une participation des citadins au développement urbain n'est pas nouvelle et s'enracine, dès les années 1980, dans un débat sur les avantages d'un développement bottom-up plutôt qu'imposé d'en haut par des gouvernements réputés bureaucratiques et les agences de coopération. Associée aux principes de recouvrement des coûts et de réplicabilité à l'échelle circonscrite des projets, constitutive de nombreux montages opérationnels, elle apparaît alors comme un volet idéologiquement acceptable d'une théorie de l'efficacité économique à destination des pauvres.

Ce constat conduit progressivement à polariser le débat autour des notions d'efficacité et d'équité. En tant que moyen, la participation serait ainsi destinée à améliorer l'efficacité de la gestion des projets et la rentabilité des investissements réalisés ; en tant que fin, elle viserait à accroître les capacités et l'autonomie des communautés, leur empowerment. La pratique, dans les villes en développement, n'a pourtant guère tiré les enseignements de cette grille analytique, la rhétorique participative mêlant généralement les deux objectifs, en dépit de nombreuses études empiriques qui attestent l'extrême difficulté à les concilier.

Dans les années 1990, les débats sur la modernisation de l'action publique et le rôle de l'État suscitent un regain d'intérêt pour la participation au développement. Celle-ci s'épanouit à la faveur du désengagement des États, de la décentralisation, de l'éclosion des partenariats public-privé, d'une pensée sur la démocratie locale qui cheville le façonnement de la gouvernance territoriale urbaine à la responsabilité des gouvernements locaux et à la participation des citadins (Blair, 2000).

À l'échelle plus fine des projets d'infrastructures, l'analyse des échecs et celle des taux décevants de rentabilité conduisent au même constat (World Bank, 1993). Elle impute ces inefficacités à la faiblesse institutionnelle des administrations africaines, dénonçant leur centralisation, leur manque de flexibilité, leur incapacité à répondre de manière appropriée aux demandes (responsiveness), leur absence de responsabilisation (accountability) envers les usagers/contribuables comme envers les bailleurs financiers.

\section{B. Une conception néolibérale de la participation}

Portée par la contestation néolibérale du rôle hégémonique de l'État dans l'énonciation et la mise en œuvre des politiques urbaines, mais aussi par les acteurs d'un monde « non gouvernemental » influent, la participation est remisée dans la pensée et la pratique du développement avec une nouvelle rhétorique. Celle-ci est adossée à quatre registres qui, diversement combinés, expliquent et justifient ses nouvelles modalités.

\footnotetext{
${ }^{2}$ Le managérialisme est ici défini comme « la croyance que tout problème politique a une solution managériale » (McCourt, 2001, p. 108),
} 


\section{Une approche consensuelle de la société}

L'approche dominante des années 1980 présentait l'État comme un frein à la croissance et le marché comme le moyen le plus efficace de promouvoir le développement économique et social dans un contexte de la mondialisation. À la fin des années 1990, après 15 ans d'ajustement structurel et de libéralisation largement infructueux, les tenants de cette stratégie ont infléchi leur discours, portant une attention plus marquée aux réformes institutionnelles et au développement social (World Bank, 1997). C'est dans ce contexte que sont « redécouvertes» les notions de société civile, de participation et de capital social ${ }^{3}$.

Pour les néo-libéraux, la société civile et ses différentes organisations doivent exercer une pression salutaire sur les appareils d'État pour consolider la démocratie et favoriser l'inclusion sociale. Une large part de la littérature récente sur le sujet s'attache ainsi à identifier et formaliser les «bonnes » techniques de participation. Cette orthodoxie participative suscite néanmoins nombre de critiques, qui soulignent sa méconnaissance des rapports de pouvoir et son format inadapté à une compréhension correcte des déterminants du changement social et technique (Cleaver, 2001). Relevant d'une stratégie descendante de réforme des institutions, les mécanismes participatifs sont en effet perçus à la fois comme des ressorts d'une efficacité accrue des institutions et comme des vecteurs d'intégration de groupes cibles dans le processus de développement. Ils supposent que le renforcement du pouvoir des dominés peut être réalisé au sein de l'ordre social existant (Mohan, Stokke, 2000). Cette conception harmonieuse des rapports de force peut dès lors se dispenser d'une analyse sérieuse des inégalités et d'une théorisation du rapport entre pauvreté et inégalités : "L'idée que la richesse des uns puisse être liée à (ou même une cause de) de la pauvreté des autres, est exorcisée d'emblée » (Lautier, 2002, p. 147).

Cette revalorisation de la participation et du local n'est cependant pas le seul apanage des néo-libéraux. Un consensus déroutant marque leur appropriation par des mouvements et acteurs appartenant à un large spectre politique, allant de la « Nouvelle gauche » néo-marxiste à la « Nouvelle droite » néo-libérale en passant par des mouvements populistes (Mohan, Stokke, 2000). Pour les néo-Marxistes par exemple, l'empowerment est affaire de mobilisation collective des groupes dominés, à la fois contre l'État et contre le marché. Se démarquant du réductionnisme marxiste, ils mettent l'accent sur la diversité des formes de domination (outre celles qui procèdent du seul rapport aux moyens de production), et soulignent la multitude des actions collectives qui peuvent en résulter. Conçues comme un instrument de lutte contre les intérêts hégémoniques, ces dernières sont d'emblée placées par les «anti-libéraux » dans un rapport d'adversité et de confrontation (Oldfield, 2003).

Les similarités sémantiques dissimulent donc de fortes divergences idéologiques, qui compliquent l'analyse critique des discours et pratiques de la participation. Nous nous en tiendrons, par la suite, à l'analyse de l'approche néo-libérale de la participation, très prégnante partout en Afrique et particulièrement hégémonique dans la réforme des services.

\footnotetext{
${ }^{3}$ Voir définition infra (note infra-paginale $\mathrm{n}^{\circ} 6$ ).
} 


\section{Du citoyen participant au stakeholder}

Chez les Anglo-saxons, la participation des usagers est souvent associée à celle du stakeholding government (Page, 2002). Empruntée au monde des entreprises, la notion renvoie, en sciences politiques, à l'idée que tout citoyen a une responsabilité dans le débat collectif, que les solutions doivent être discutées et négociées avec l'ensemble des «parties prenantes ». Cette conception du gouvernement valorise des dispositifs de partage du pouvoir et de négociation des décisions, ouvrant sur des interrogations relatives aux voies et moyens de la participation.

Dans les services d'eau, l'adoption de cette approche induit l'élargissement des procédures de participation à tous les stakeholders (autorités organisatrices, opérateurs, bailleurs financiers, usagers...), que la formalisation de relations contractuelles tente de canaliser au profit d'un petit nombre de figures prévisibles et reconnaissables (Cleaver, 2001, p. 40). La formation d'associations d'usagers légalement reconnues est ainsi partout encouragée, soit pour agir comme délégataire principal d'une installation ou d'un ensemble d'installations (dans le modèle du comité exploitant), soit pour servir d'instance de régulation de proximité et de représentation des usagers (dans le modèle de la délégation à un tiers). Plusieurs États d'Afrique de l'Ouest ont ainsi modifié leur législation, ou envisagent de le faire, pour permettre l'organisation de ces associations, leur confier la responsabilité de la fourniture du service et éventuellement leur transférer la propriété des installations (Étienne, 1998 ; Coing et al., 1998). Ailleurs, des formules hybrides tentent aussi d'associer la gestion déléguée des mini-réseaux d'adduction d'eau à une régulation communautaire. L'objectif est de forger, à une échelle appropriée, des institutions capables de gérer le patrimoine commun d'un collectif d'usagers et d'offrir à celui-ci un canal d'expression et de représentation dans les aires urbaines où le réseau conventionnel se révèle, provisoirement ou durablement, inadapté.

Cette dévolution de la fourniture à des entités infra-nationales entérine le fait que les pouvoirs publics, dans ces sociétés, ne détiennent pas le monopole de l'organisation des services. Dans les arrangements qu'elle promeut, elle présuppose néanmoins la fonctionnalité de processus d'accommodement entre des intérêts variés, ceux des usagers - qui ne constituent pas un ensemble homogène - aussi bien que ceux des opérateurs privés, formels et informels, subsumant leurs antagonismes dans une commune appropriation du service.

\section{La subsidiarité}

La participation est souvent confondue avec la décentralisation, c'est-à-dire la dévolution du pouvoir de décision à des instances proches des populations, au prétexte que la seconde favorise généralement la première (Tunstall, 2001). Tout en réaffirmant ce lien, la Banque mondiale admet néanmoins que, pour l'instant, les résultats de la décentralisation "vue comme un moyen de permettre la participation populaire » demeurent ténus (Decentralization Thematic Team-a, s.d.).

L'Afrique urbaine offre ainsi de nombreuses illustrations des multiples figures de la déconnexion entre décentralisation proclamée et processus participatifs, tôt décrites dans l'émergence de «systèmes mixtes d'administration locale » (Mawhood, 1987) puis décortiquées dans nombre d'études postérieures. Ainsi, comparant plusieurs pays dont deux en Afrique de l'Ouest (Ghana et Côte d'Ivoire), Richard Crook et Alan Sverrisson ne parviennent pas à établir une relation probante entre décentralisation et 
responsabilisation, notamment à l'égard des pauvres (Crook, Sverrisson, 2001). Au Zimbabwe et en Tanzanie, Arild Schou souligne que la pénurie d'argent public et les médiocres compétences des agents et politiciens « de proximité » annulent les effets attendus en matière d'efficacité ${ }^{4}$ (Schou, 1999). Là où la décentralisation a induit une redistribution des responsabilités et des ressources financières, la démocratie de représentation, dans des sociétés très inégalitaires, a souvent été confisquée par des élites locales (Jaglin, Dubresson, 1993). Plus généralement, dans des États patrimoniaux où les mécanismes de contrôle peinent à s'imposer, l'accès aux services demeure souvent contrôlé par des filières clientélistes, aux échelles locales comme nationales (Goetz, Gaventa, 2001, p. 13).

Modifiant l'angle d'analyse, l'accent est aujourd'hui davantage placé sur les processus locaux d'organisation (plutôt que de dévolution de fonctions centrales), suscitant un nouvel intérêt pour le principe de subsidiarité (World Bank, 1993, p. 15). Élargie à l'ensemble des parties concernées (stakeholders) et organisée à l'échelle de la plus grande proximité possible, la participation est alors présentée, dans les services d'eau, comme de nature à relever trois défis :

- Faire émerger la demande des populations, en s'appuyant notamment sur des " experts locaux». La participation doit faciliter l'expression et la formulation de cette expertise profane et, éventuellement, la défense (advocacy) des demandes qu'elle révèle.

- Promouvoir une méthode itérative d'essais-erreurs. Reconnaissant que de nombreux échecs sont liés à l'inadaptation des solutions importées, les nouvelles approches s'appuient sur la participation pour forger cette adéquation par une méthode itérative d'essais-erreurs (Subramanian et al., 1997, p. 100).

- Mobiliser des «communautés d'intérêts» au service d'une gestion durable. La participation doit permettre d'assurer l'implication, sur le long terme, de l'ensemble des stakeholders dans un système de gestion local, fondé sur l'action concertée et la négociation des équilibres contributions/rétributions.

\section{De la décentralisation politico-administrative à la décentralisation économique}

Les approches en termes de gouvernance ont enfin un rôle essentiel dans les mutations de la participation. Insistant sur la nécessité de reléguer l'Etat dans les modes de coordination entre acteurs qui, à l'échelle locale, concourent à l'élaboration des conventions collectives, bailleurs et experts légitiment des dispositifs de gouvernance impliquant, "à côté des institutions publiques, elles-mêmes avec des nouvelles fonctions, les autres acteurs locaux [...], que ce soit les organisations professionnelles ou de représentation, le secteur privé, et autres organisations non gouvernementales » (Decentralization Thematic Team-a, s.d.). Cette évolution est très marquée dans les services d'eau, dont la gestion est encadrée par de nouveaux principes, d'inspiration néo-libérale, synthétisés en 1992 à la conférence de Dublin (World Bank, 1993, p. 24).

Autour des enjeux de gouvernance qui s'ensuivent, la conception politique et administrative de la décentralisation, qui visait à responsabiliser les opérateurs publics locaux et à améliorer leurs performances, cède progressivement le pas (en termes de priorité des réformes) à une conception économique de la décentralisation. Celle-ci

\footnotetext{
${ }^{4}$ En termes d'adéquation de la gamme des services aux préférences des usagers et d'efficacité productive (quantité de services produits en fonction des moyens mis en œuvre).
} 
prône désormais le démembrement du secteur public ${ }^{5}$ et la construction d'un marché ou d'un quasi marché - de services.

Au-delà des affichages, le sens et le contenu des enjeux de participation s'en trouvent profondément modifiés. Dans la décentralisation politique et administrative, la participation devait réconcilier les usagers-citoyens avec des autorités publiques démocratisées, prestataires de services collectifs. Dans la décentralisation économique, la perspective est tout autre : il s'agit de mobiliser les «organisations de la société civile », vague notion englobant les usagers et leurs organisations aussi bien que les petits entrepreneurs informels et les industriels de l'eau, dans de nouveaux partenariats public-privé. En fonction de la distribution des rôles, la participation des uns et des autres peut aussi bien renvoyer à la notion de paiement (pour le consommateur individuel), qu'à celle d'organisation (pour les « communautés » impliquées dans la coproduction des services) et de partenariat (pour les artisans et entrepreneurs privés). Il s'ensuit une grande confusion du terme de participation, que nous préférons réserver aux modalités d'intervention des usagers dans la gestion et la régulation des services.

Couplée et subordonnée à la décentralisation économique, la participation ainsi définie vise moins à favoriser la démocratie délibérative et participative qu'à «doper » l'efficacité de l'action publique et collective par des mécanismes de quasi marché, en conformité avec les principes du New Public Management. Dépolitisée, elle est consignée aux échelles de proximité, dans un espace économique fonctionnel local dont la décentralisation et la participation accroissent les performances économiques.

\section{ENTRE RHETORIQUE ET PRATIQUES : PETITES ET GRANDES AMBITIONS DES DISPOSITIFS PARTICIPATIFS DANS LES SERVICES D'EAU}

Après vingt ans de réformes des services d'eau, le bilan n'est guère probant. Les échecs sont en partie interprétés comme résultant d'une crise de confiance des citoyens dans des appareils d'État réputés corrompus, arrogants et insensibles aux demandes spécifiques.

Cette défiance provient d'abord des pauvres, dont l'insatisfaction est étroitement liée à l'absence de mécanismes efficaces d'expression de leurs préférences (voice options) et de responsabilité des autorités à leur égard (Narayan et al., 2000). À l'autre bout de l'échelle sociale, la contestation n'est pas moindre : elle porte sur l'uniformité de l'offre et les dysfonctionnements des services ; elle s'enracine aussi, de façon plus ou moins explicite, dans un refus des solidarités automatiques dont les péréquations tarifaires étaient une manifestation. Ces demandes des couches moyennes et supérieures, intégrées dans la mondialisation et aspirant à des normes de service et de consommation internationales, supportent les politiques visant à accroître la compétitivité urbaine et sont exprimées de manière aussi vindicative que celles des pauvres, contraignant les gouvernements urbains à de difficiles arbitrages.

\footnotetext{
${ }^{5}$ Ainsi, pour la Banque mondiale : «Les formes de décentralisation les plus complètes, du point de vue du gouvernement, sont la privatisation et la dérégulation car elles transfèrent les responsabilités des fonctions administratives du secteur public au secteur privé [...]. Elles permettent que des fonctions qui relevaient primordialement ou exclusivement du gouvernement soient exercées par le secteur privé, les communautés, les coopératives, les associations volontaires privées et d'autres organisations non gouvernementales » (Decentralization Thematic Team-b, s.d.).
} 
Pour dépasser cette crise de confiance, les politiques participatives sont organisées autour de deux enjeux : la protection du consommateur et la co-production des services.

\section{A. Participation et responsabilité : le consommateur triomphant}

L'objectif est ici de regagner la confiance de l'usager, ce à quoi ne sont pas parvenues les réformes de décentralisation. La réflexion s'organise autour d'une refonte des liens entre participation et mécanismes horizontaux de responsabilisation (Blair, 2000). Dans les réseaux techniques locaux, elle pourrait conduire à associer les usagers à la régulation des services, au prix d'une timide remise en cause du monopole de l'État sur la surveillance et la sanction de ses agents (Goetz, Gaventa, 2001, p. 8).

En Afrique comme ailleurs, cette approche s'inscrit dans une modernisation des services publics très influencée par le New Public Management (McCourt, Minogue, 2001). Partisans d'un «meilleur gouvernement », plus performant et moins coûteux, ses théoriciens soutiennent la mise en œuvre d'un ensemble cohérent de mesures propres à galvaniser l'efficacité et la performance des services. Parmi celles-ci se trouve la participation des usagers qui, en introduisant des mécanismes de quasi-marché dans les systèmes de fourniture, permettrait d'en accroître la qualité et la réactivité.

L'influence du New Public Management est partout sensible en Afrique subsaharienne, mais les expériences demeurent limitées et les études d'évaluation très dispersées (McCourt, 2001). Les accords de performance incluant la production d'indicateurs, de mesures et de normes de qualité sont aujourd'hui assez répandus, même si leur pertinence est souvent discutée, tandis que les principaux outils utilisés pour accroître la qualité et la réactivité des services sont les enquêtes d'opinion et de satisfaction, les chartes de consommateur, la formalisation de procédures de réclamation. On en trouve des exemples dans les grandes villes sud-africaines, notamment au Cap.

L'usage de ces techniques de marketing dans la régulation des services pose cependant un certain nombre de problèmes. Le premier est qu'il concerne uniquement les citadins desservis, les autres, souvent majoritaires, étant par définition exclus de la relation de service. Il n'est donc pas certain que des outils de type enquêtes de satisfaction puissent alimenter un débat public démocratique, du moins dans les conditions actuelles de leur utilisation. Ensuite, la représentation des usagers, dont le poids varie en fonction des quantités et de la qualité des services consommés, donc des pouvoirs d'achat, tend à favoriser les « gros consommateurs », domestiques et commerciaux. Plus généralement, on note une sur-représentation des couches moyennes et de leurs intérêts dans l'ensemble des dispositifs participatifs.

Le lobbying via des associations ou des organisations communautaires et, éventuellement, le recours aux tribunaux, autre moyen de peser sur les politiques de service, est potentiellement plus ouvert aux publics défavorisés représentés par des ONG et des organisations de défense de leurs intérêts. En Afrique du Sud, de récents procès concernant par exemple le droit à l'eau (et au logement) ont interprété de manière avantageuse pour les pauvres les principes constitutionnels. On n'est guère surpris, néanmoins, de constater que les couches moyennes sont, là comme ailleurs, plus habiles à défendre leurs intérêts (Beall, Crankshaw, Parnell, 2002, p. 200).

De plus, dans les quartiers aux populations pauvres et vulnérables, ces formes de participation parviennent à peine à entamer l'hégémonie d'un autre modèle, celui de la co-production, qui en éclipse voire en vampirise les dynamismes. Certes, Sophie 
Oldfield montre que, dans certains quartiers du Cap, des organisations parviennent à combiner un dialogue continu avec les employés des services de police, de santé ou de logement, afin d'orienter leurs pratiques (responsiveness), et des modes d'action plus revendicatifs et contestataires (Oldfield, 2003, p. 14). Toutefois, une telle situation n'est possible qu'en présence de services publics raisonnablement efficaces, ce qui n'est pas une situation générale dans les villes d'Afrique subsaharienne. Lorsque l'enjeu est de créer ou d'universaliser les services, la pérennité d'arrangements composites tels que ceux décrits au Cap est plus problématique, les dispositifs de coproduction ayant tendance à accaparer les ressources, par définition limitées, des communautés défavorisées.

\section{B. Participation et diversité des demandes : les pauvres dans la co-production des services}

Le second axe de réflexion insiste sur l'inadaptation d'une conception des services publics fondée sur une offre uniforme et normée. Il n'est pas spécifique aux pays en développement et s'inscrit dans un double mouvement de reconnaissance des publics hétérogènes et de différenciation de l'offre. Le poids des exclus du service officiel modifie toutefois profondément, dans les villes africaines, le sens de cette diversification : affectant des villes dont en moyenne $40 \%$ des citadins sont des orphelins du réseau, elle sous-tend de formidables enjeux matériels et financiers et mobilise des formes spécifiques de participation.

Les expériences africaines sont ici nombreuses (Jaglin, 2000 ; Lyonnaise des Eaux, 1999). Elles sont principalement motivées par la question de l'universalisation en contexte de grande pauvreté et s'appuient sur trois « piliers ». Premièrement, l'abandon, dans les législations nationales, de l'objectif d'une généralisation du service (le « robinet à domicile pour tous ») au profit de celui d'une universalisation de l'accès à l'eau potable, compatible avec une différenciation du service. Deuxièmement, la systématisation de l'approche-client appuyée sur la mise en œuvre d'outils révélant les préférences des consommateurs (enquêtes de capacité/volonté de payer pour un niveau de service déterminé). Enfin l'externalisation, auprès d'usagers organisés, d'une partie des coûts d'infrastructure et de gestion.

C'est ce dernier avatar que nous qualifions ici de co-production des services d'eau. Les formes d'organisation en demeurent diverses et inégalement élaborées (accord oral avec un « représentant communautaire », comité de point d'eau, association d'usagers formelle) mais l'objectif d'encadrement des dynamiques participatives y est aujourd'hui explicitement associé à celui d'une extension du modèle marchand. Avec le principe du financement par le tarif, la participation se trouve ainsi rapatriée dans le noyau dur des complexes réformateurs.

Loin d'être isolée, cette ingénierie participative témoigne, dans les pays en développement, d'un regain d'intérêt plus général pour les institutions d'échelle communautaire, les normes informelles et les réseaux interpersonnels susceptibles de nourrir une action collective efficace et durable, notamment dans la gestion locale des ressources et biens communs (Evans, 1996). Quelques études portent plus spécifiquement sur les conditions d'apparition et de consolidation de normes de coopération entre des opérateurs publics et des organisations de citadins (Ostrom, 1996 ; Tendler, 1997), en soulignant le rôle qu'y jouent des arrangements 
institutionnels, formels et informels, fondés sur une utilisation intensive d'information, de confiance et de capital social ${ }^{6}$ (Pamuk, 2000).

La «petite dimension» privilégiée dans ces travaux est une notion sociologique (la «communauté ») plus que géographique. Dans le contexte de sociétés aux cadres d'action juridiques et réglementaires mal stabilisés, caractérisés par un fort degré d'incertitude ou fragilisés par un déni de légitimité, cette dimension " communautaire » séduit. Elle permet d'envisager la reconstruction de capacités d'action collective de modeste envergure à l'échelle d'espaces pertinents, à forte dimension cognitive, où peuvent être plus aisément mobilisées des ressources endogènes composites, intériorisés des savoirs et savoir-faire spatialisés, réconciliés les intérêts de groupes qui partagent l'usage et le contrôle des lieux. Valorisant des normes et règles partagées, recourant à des mécanismes de sanction gradués, la gestion à l'échelle communautaire rendrait aussi difficile, pour tout individu ou groupe, de se soustraire aux règles communes de la réciprocité et limiterait le free-riding.

Cette démarche est aujourd'hui très présente dans la réflexion et les projets sur les services urbains des villes d'Afrique subsaharienne. Réplique aux échecs de nombreux projets « détournés » ou rejetés, elle mise sur les réseaux sociaux locaux pour construire entre opérateurs et usagers des conventions indispensables à la durabilité du service d'eau en présence d'intérêts divergents. Admettant que convention et confiance ${ }^{7}$ se renforcent mutuellement et se trouvent facilitées lorsqu'elles s'enracinent dans un socle de références et de règles de vie partagées, dont on présume qu'elles diffèrent d'un lieu à l'autre (et selon la position relative des individus et des groupes dans chacun), cette démarche présente la relocalisation des dispositifs de fourniture à des échelles mieux maîtrisées comme un moyen d'élaborer et de légitimer des cadres d'action collective dans des sociétés urbaines hétérogènes.

Toutes ces expériences s'accompagnent d'un effacement variable des encadrements publics, au profit d'une affirmation des pouvoirs locaux «communautaires », en conformité avec certaines hypothèses néo-institutionnalistes sur l'administration coloniale héritée, qui n'aurait pas de base juridique et politique intériorisée (Hibou, 1998, p. 15), et l'importance des relations de confiance et de pouvoir dans le fonctionnement d'institutions informelles efficaces. On en a inféré un peu vite que celles-ci seraient favorisées, aux échelles locales, par un rôle accru des pouvoirs «communautaires ». De fait, en raison de leur légitimité et de leur autorité locales, ceux-ci assument souvent un rôle d'encadrement. Leur habileté peut temporairement masquer le déficit de régulation officielle, mais elle suffit rarement à établir un système fiable et stable de garantie et de recours, même à l'échelon du voisinage, et elle aggrave la compétition inter- et intra-« communautés » pour l'accès à des ressources limitées. Les gestionnaires locaux sont alors souvent démunis face aux situations de détresse que ne prennent pas en charge les dispositifs de la gestion de proximité mais dont ils ont à

\footnotetext{
${ }^{6}$ Le capital social est ici défini comme une ressource issue des interactions entre agents, du développement endogène de normes de comportement et de l'institutionnalisation de ces normes en règles. Fondé sur des réseaux sociaux, des normes de réciprocité (ou de confiance), des valeurs et convictions communes, il est susceptible de générer des externalités durables qui affectent la situation économique des individus et groupes, notamment en facilitant l'action collective.

${ }^{7}$ Nous faisons ici référence à la notion de confiance telle qu'elle apparaît depuis quelques années dans la littérature (Bernoux, Servet, 1997), mettant en jeu des éléments comme la croyance dans l'autre et dans la validité des engagements pris (qu'ils reposent sur un document écrit ou non), la légitimité des règles, la mémoire et l'expérience partagées...
} 
affronter les manifestations quotidiennes : les squatters ont-ils le droit de prendre de l'eau aux robinets collectifs à Windhoek alors qu'ils ne peuvent être officiellement facturés ? Les gérants privés de bornes-fontaines, autorisés à de justes (fair) bénéfices par leur contrat de gérance, peuvent-ils augmenter leurs prix auprès des usagers payants pour faire face à la demande d'eau gratuite des indigents à Lusaka ? Sans compter les barons et autres «mafias » locales, pour qui l'eau n'est qu'une des ressources possibles de la construction de pouvoirs territoriaux en ville.

Les régulations «communautaires», en ville, ne suffisent pas à un «bon» fonctionnement des services. À Ouagadougou, la gestion partagée des points d'eau collectifs a accru les inégalités socio-spatiales dans les périphéries (Jaglin, 1994). À Windhoek, propriétaires, locataires et squatters s'affrontent autour des robinets publics (Peyroux, 2000). En Afrique du Sud, la ville de Durban constate que, dans les quartiers pauvres, la stabilité des communautés et des comités de gestion est insuffisante pour permettre le développement d'un réseau de bornes-fontaines publiques (Urban Management, November 1997, p. 36). À Lusaka, les Residents Development Committees se heurtent à des problèmes récurrents de fiabilité du fonctionnement des kiosques à eau (Bousquet, 2004). Plus généralement, on peut s'interroger sur la pertinence, à moyen terme, d'un dispositif dit « communautaire » pour assurer la gestion des mini-réseaux d'adduction d'eau urbains (Coing et al., 1998 ; Manor, 2002).

Une partie du déficit de la réflexion provient de ce que les théories de l'action collective n'ont pas de modèles propres aux sociétés urbaines, dont elles méconnaissent les spécificités. Appliquées à l'eau, les analyses s'intéressent surtout aux communautés rurales, à la gestion de la ressource et à l'irrigation plutôt qu'à l'approvisionnement des ménages (Ostrom, 1992 ; Cleaver, 2000). Leur transposition aux milieux urbains (Subramanian et al., 1997, p. 104) repose sur une conjecture, celle qu'existent en ville des «communautés d'intérêt » stables, porteuses d'enjeux collectifs et correctement informées, qu'elles sont une bonne échelle de gestion des services et ont les moyens d'exprimer leurs préférences, qu'un système de responsabilisation incite politiciens et personnels administratifs à leur être attentifs... Autant de conditions pourtant rarement remplies dans les villes africaines. Beaucoup de ces analyses ont aussi fait l'objet de critiques sur leur conception a-historique, statique et souvent simpliste des relations sociales et des réalités locales (Cleaver, 2001), indifférente à la multiplicité et à l'ambivalence des répertoires culturels (Hibou, 1998, p. 15). En insistant sur le capital social comme ressource de l'action collective, elles négligent par exemple ses externalités négatives dans des sociétés inégalitaires et hiérarchiques (exclusion sociale) ou certaines de ses formes perverses (mafia). Enfin, le principal problème des sociétés urbaines africaines ne semble pas être la densité des liens et l'intensité de la confiance à l'échelle micro-locale, mais bien les difficultés constatées à déployer ce capital social à des échelles où l'action politique et économique devient efficace (Evans, 1996, p. 1124 ; Tostensen et al., 2001).

\section{PARTICIPATION ET INCLUSION SOCIALE : UNE FAUSSE EVIDENCE}

L'unanimisme des discours sur la participation masque ainsi la diversité des pratiques, socialement très marquées. Pour les clientèles solvables, la « décentralisation » passe par le marché et la participation s'exprime dans le cadre d'un code formalisé des consommateurs, visant à leur assurer des droits, une protection, un choix. Là où la 
libéralisation risque d'engendrer un déficit d'offre, notamment dans les aires peu rentables (quartiers pauvres des centres-villes, périphéries à faibles densités, petites villes, zones rurales), la participation est davantage synonyme d'une co-production des services. Par la mobilisation monétaire et matérielle des ménages, elle vise surtout à favoriser la co-construction des infrastructures et la co-gestion de réseaux décentralisés.

A priori, ces modes de participation et leurs objectifs ne sont ni exclusifs ni contradictoires, dans la pratique pourtant, ils apparaissent spécialisés en fonction des publics. Aux uns, les outils progressivement formalisés d'un consumérisme régulé ; aux autres, les voies alternatives de la mobilisation, conduisant à une intégration, au rabais, dans les circuits marchands des services. Entre une participation des « riches » dépolitisée, constitutive de la réforme managériale des pouvoirs locaux, et la participation des pauvres, les convergences ne sont guère apparentes. Il n'est nullement nécessaire de conclure à un mouvement délibéré. Beaucoup plus probablement, ce résultat est déterminé par le contexte idéologique dans lequel s'insèrent les dispositifs participatifs d'une part, l'économie politique des réformes d'autre part.

\section{Participation et marché : une propédeutique à l'usage des pauvres}

Ces dispositifs participatifs répondent-ils mieux aux demandes urbaines et, particulièrement, à celles des populations déshéritées ? Il n'est pas utile ici de revenir sur la participation des consommateurs dans des dispositifs «privatisés » ou corsetés par les principes du New Public Management qui apparaît, dans sa conception même, comme un des ressorts de la régulation de dispositifs d'approvisionnement en eau « marchandisés ». Qu'en est-il en revanche des systèmes de co-production, modalité principale pour ne pas dire exclusive de la participation des pauvres ?

Le bilan quantitatif demeure mal connu mais il est loin d'être négligeable. Il est aujourd'hui largement admis qu'une part croissante des ménages urbains dépendra, en Afrique, de dispositifs de desserte non conventionnels mobilisant, selon des modalités et à des degrés divers, les populations et leurs organisations. Incontestablement, cette ingénierie participative, conditionnalité de nombreux financements extérieurs, participe d'une universalisation de l'accès à l'eau potable et peut contribuer à une amélioration, lente et inégale certes, des conditions matérielles de vie en ville.

Favorise-t-elle pour autant une meilleure représentation des intérêts des pauvres ? Remarquons que leur intégration urbaine est réalisée au prix d'une diversification technico-gestionnaire et sur la base de représentations et comportements qui lient étroitement les notions de responsabilité individuelle et collective à celle de paiement ${ }^{8}$. Fournissant un support à l'apprentissage du user-pays system et la justification d'un abandon des anciennes politiques sociales de l'eau, réputées inefficaces, elle vise la durabilité économique des systèmes locaux de desserte et fait entrer les pauvres dans le marché plus sûrement que dans la démocratie.

Aussi n'est-elle pas instigatrice d'une conception renouvelée de la question sociale. Loin de contredire les logiques commerciales, la participation est un outil de régulation/stabilisation des nouveaux dispositifs. Elle facilite un compromis entre la reconnaissance du droit de disposer d'un volume d'eau potable minimal (ce que recouvre la notion internationale d'accès à l'eau potable) et le principe présenté comme

\footnotetext{
${ }^{8}$ Ben Page en fournit une très belle illustration à travers l'exemple du service d'eau de Kumbo au Cameroun (Page, 2003).
} 
intangible de la rémunération du service par le tarif. Dans les quartiers pauvres, la participation des habitants est la traduction privilégiée de ce compromis.

La démarche tient à une idée simple mais fondatrice : pour les bailleurs de fonds et principaux mentors des institutions de développement, «la pauvreté a pour origine fondamentale la difficulté ou l'incapacité des pauvres à accéder au marché » (Lautier, 2002, p. 140). Les aider, c'est leur conférer des capabilities et des opportunités d'organisation au sein de la société dite civile pour y parvenir. Universaliser l'accès à l'eau potable dans le cadre d'une relation marchande clairement établie semble y contribuer. Mais l'enjeu est-il univoque?

Il est notoire que des cohortes significatives de citadins sont, en Afrique subsaharienne, exclues des marchés formels, légaux, du travail, du sol et du logement, des services... toutefois, elles s'inscrivent dans un réseau dense de relations et filières marchandes informelles, dynamiques et diversifiées. Disqualifier ou formaliser ces dernières au profit de dispositifs participatifs encadrés n'assure donc pas l'insertion des pauvres dans le marché, mais dans sa sphère officielle, régulée par les pouvoirs institutionnels. En d'autres termes, ne s'agit-il pas aussi d'améliorer la «traçabilité » (notamment fiscale, grand enjeu des réformes de décentralisation) et le contrôle des filières d'approvisionnement en eau ? Qui cherche-t-on dès lors vraiment à aider ?

\section{Une économie politique des réformes}

Comment expliquer une telle perméabilité des sociétés urbaines africaines aux visions étroitement managériales de la gestion participative ? Pourquoi un tel consensus en faveur de politiques qui s'attaquent certes aux dysfonctionnements des réseaux, en contribuant à doper la réactivité des opérateurs ici, à étendre les infrastructures là, mais au prix de nouvelles inégalités socio-spatiales ?

Il faut d'abord rappeler que la stabilité politique des sociétés urbaines africaines a reposé, depuis les indépendances, sur une alliance entre pouvoirs d'État et couches moyennes, à laquelle la gestion publique subventionnée des services publics a amplement contribué : l'énonciation d'un droit consumériste pour les usagers des réseaux renouvelle en la réaffirmant cette alliance. Celle-ci peut, certes, être localement minée par l'appauvrissement voire l'érosion des couches moyennes, durement frappées par les mesures d'ajustement structurel, mais l'introduction d'acteurs privés en a modifié la logique de reproduction, en l'élargissant aux couches urbaines solvables, y compris pauvres. En visant ces «nouvelles » cibles commerciales, les entreprises concessionnaires procurent aux rapports de pouvoir dominants le lubrifiant nécessaire à leur reproduction. Ainsi, elles contribuent à diffuser et à légitimer les normes marchandes et les pouvoirs qui s'en font les promoteurs, contrant la menace que, dans de nombreux pays, la fragilisation des couches moyennes urbaines exerce sur la durabilité des services.

Ce faisant, les systèmes réformateurs repoussent, tout en la réactivant, la démarcation avec d'autres citadins, les pauvres «structurels ». Ceux-là tendent certes aussi à l'intégration dans le marché, mais dans l'enclos communautaire endogéré. En déplaçant le débat vers le renforcement du pouvoir des pauvres (empowerment), la gouvernance participative conduit à s'interroger sur les moyens à leur donner pour s'extraire de la misère, sans remettre en cause les inégalités existantes. En d'autres termes, comme ironise Bruno Lautier, « Le pouvoir des pauvres, c'est d'abord à eux de se le donner, et 
ils n'auront de pouvoir que sur eux-mêmes » (Lautier, 2002, p. 158). Participation et empowerment peuvent, dans le strict cadre de la discipline marchande, améliorer l'accès des pauvres à certaines prestations, pour peu qu'ils soient organisés au sein de communautés «vertueuses et fonctionnelles »(Chipkin, 2003). La place qui leur est ainsi conférée, au sein de dynamiques collaboratives, par l'approche managériale et techniciste de la gestion urbaine laisse toutefois entières les questions d'expression des pauvres dans le champ politique, donc leur capacité à influencer les politiques urbaines.

\section{CONCLUSION}

Outre qu'elle «neutralise » certains des effets déstabilisateurs de la pauvreté, la coproduction dévitalise des formes plus contestataires d'organisation. La montée du consumérisme légitime les normes marchandes auxquelles aspirent les couches moyennes. Combinés, ces modes de participation des usagers dans les services d'eau sont peu efficaces comme ressorts d'une gouvernance urbaine démocratique et n'influencent que marginalement les inégalités socio-spatiales. Ils fonctionnent en revanche comme une régulation de la reproduction des appareils politiques dominants. Ceux-ci n'ont bien souvent ni la capacité ni la volonté de penser, encore moins de traiter les tensions résultant de la marchandisation des services. S'ils s'accommodent du droit d'expression et de la reconnaissance de ceux qui en pâtissent le plus, on est loin d'un partage du pouvoir et rares sont les dispositifs participatifs adossés à de véritables stratégies redistributives, leviers de politiques sociales au design renouvelé. En l'absence d'une définition générale de la participation des usagers, politiquement sensible, les réformes se satisfont de la formalisation de mécanismes participatifs ad $h o c$, en fonction des problèmes soulevés par les divers segments de clientèle.

Une posture critique à l'égard de la participation ne doit donc pas seulement questionner les moyens et procédures mis en œuvre, ni même leurs incidences sur la substance de certaines décisions, elle doit aussi en analyser l'économie politique et la place dans les régulations à l'échelle locale. En l'occurrence, si les services urbains peuvent contribuer à réduire les fractures socio-spatiales engendrées par le marché, comme feignent de le croire les décideurs nationaux et internationaux, ce ne peut être que sur la base de leur universalisation, laquelle requiert des choix de société et des décisions radicales en termes d'allocation des ressources financières. Or, la participation, telle qu'elle est aujourd'hui déployée, ne permet pas de peser en ce sens. Raffermissant, là, des liens dégradés avec certaines catégories d'usagers, nouant, ailleurs, une relation avec des exclus du réseau, les mécanismes et procédures mobilisés la cantonnent dans des tâches managériales et techniques. Ce n'est pas négligeable mais néanmoins insuffisant pour rapatrier les questions d'équité socio-spatiale au cœur des réformes néo-libérales. C'est donc d'ailleurs que devra provenir la réflexion sur les voies et moyens de l'universalisation des services d'eau en Afrique subsaharienne.

\section{RÉFÉRENCES BIBLIOGRAPHIQUES}

BANQUE MONDIALE, 1994, Rapport sur le développement dans le monde : une infrastructure pour le développement, New York, Oxford University Press, 268 p.

BEALL Jo, CRANKSHAW Owen, PARNELL Susan, 2002, Uniting a Divided City : Governance and Social Exclusion in Johannesburg, London, Earthscan Publications, 237 p. 
BERNOUX Philippe, SERVET Jean-Michel (dirs), 1997, La construction sociale de la confiance, Paris, Association d'économie financière/Montchrestien, 486 p.

BLAIR Harry, 2000, «Participation and Accountability at the Periphery : Democratic Local Governance in Six Countries », in World Devlopment, vol. 28, n 1, pp. 21-39.

BOUSQUET Anne, 2004, «Desserte collective des quartiers pauvres en Zambie: un long apprentissage », in Flux, n 56-57, avril-septembre, à paraître.

CHIPKIN Ivor, 2003, "'Functional' and 'Dysfunctional' Communities : the Making of National Citizens », in Journal of Southern African Studies, 29(1), pp. 63-82.

CLEAVER Frances, 2000, «Moral Ecological Rationality, Institutions and the Management of Common Property Resources », in Development and Change, vol. 31, pp. 361-383.

CLEAVER Frances, 2001, «Institutions, Agency and the Limitations of Participatory Approaches to Development », in COOKE Bill, KOTHARI Uma (eds.), Participation : the New Tyranny?, London, New York, Zed Books, pp. 36-55.

COING Henri et al., 1998, Analyse comparative des performances de divers systèmes de gestion déléguée des points d'eau collectifs, Paris, BURGÉAP/ALFA, avril, pag. mult.

COOKE Bill, KOTHARI Uma (eds.), 2001, Participation : The New Tyranny?, London, New York, Zed Books, 207 p.

CROOK Richard C., SVERRISSON Alan S., 2001, Decentralisation and poverty-alleviation in developing countries : a comparative analysis or, is West Bengal unique?, Brighton, Institute of Development Studies, 60 p. (IDS Working Paper 130).

DECENTRALIZATION THEMATIC TEAM-a, s.d., « Historique de la Decentralisation », Washington, Banque mondiale (www.ciesin.org/decentralization/French/General/historique.html).

DECENTRALIZATION THEMATIC TEAM-b, s.d., «Qu'est-ce que la décentralisation ? , Washington, Banque mondiale (www.ciesin.org/decentralization/French/General/Qu'estceque.html).

ÉTIENNE Janique, 1998, Formes de la demande et modes de gestion des services d'eau potable en Afrique subsaharienne : spécificité des “ milieux semi-urbains ”, Paris, ENPC, 299 p. + annexes (Thèse de doctorat).

EVANS Peter, 1996, «Government Action, Social Capital and Development : Reviewing the Evidence on Synergy », in World Development, vol. 24, n 6, pp. 1119-1132.

GOETZ Anne-Marie, GAVENTA John, 2001, Bringing citizen voice and client focus into service delivery, Brighton, Institute of Development Studies, 63 p.

HIBOU Béatrice, 1998, Économie politique du discours de la Banque mondiale en Afrique subsaharienne : du catéchisme économique au fait (et méfait) missionnaire, Paris, CERI, $46 \mathrm{p}$. (Les Études du CERI, n 39, mars).

JAGLIN Sylvy, 1994, «Why mobilise town dwellers ? Joint management in Ouagadougou (19831990) », in Environment and Urbanization, vol. 6, n² 2, October, pp. 111-132.

JAGLIN Sylvy, 2000, «L'eau potable dans les villes en développement : les modèles marchands face à la pauvreté », in Revue Tiers Monde, t. XLII, n 166, avril-juin, pp. 275-303.

JAGLIN Sylvy, 2003, Réseaux et fragmentation urbaine: services d'eau en Afrique subsaharienne, Paris, Université Paris I-Panthéon Sorbonne, 232 p. (mémoire d'habilitation à diriger des recherches).

JAGLIN Sylvy, DUBRESSON Alain, 1993, Pouvoirs et cités d'Afrique noire. Décentralisations en questions, Paris, Karthala, 308 p.

LAUTIER Bruno, 2002, «Pourquoi faut-il aider les pauvres? Une étude critique du discours de la Banque mondiale sur la pauvreté », in Revue Tiers Monde, t. XLIII, n 169, janvier-mars, pp. 137165. 
LYONNAISE DES EAUX, 1999, Solutions alternatives à l'approvisionnement en eau et à l'assainissement conventionnels dans les secteurs à faibles revenus, Paris, Suez Lyonnaise des Eaux, $161 \mathrm{p}$.

MANOR James, 2002, « Partnerships between Governments and Civil Society for Service Delivery in Less Developed Countries : Cause for Concern », presented at the World Development Report 2003/04 Workshop : Making Services Work for Poor People, Oxford, 4-5 November 2002, 8 p.

MAWHOOD Philip, 1987, «Decentralization and the Third World in the 1980s », in Planning and Administration, vol. 14, $\mathrm{n}^{\circ}$ 1, Spring, pp. 10-22.

McCOURT Willy, 2001, «The NMP agenda for service delivery : a suitable model for developing countries ? », in McCOURT Willy, MINOGUE Martin (eds.), The Internationalization of Public Management : Reinventing the Third World State, Cheltenham (UK) ; Northampton (USA), Edward Elgar, pp. 107-128.

McCOURT Willy, MINOGUE Martin (eds.), 2001, The Internationalization of Public Management : Reinventing the Third World State, Cheltenham (UK) ; Northampton (USA), Edward Elgar, 265 p.

MOHAN Giles, STOKKE Kristian, 2000, « Participatory development and empowerment : the dangers of localism », in Third World Quaterly, vol. 21, n² 2, pp. 247-268.

NARAYAN Deepa et al., 2000, Voices of the Poor : crying out for change, Washington, The World Bank.

OLDFIELD Sophie, 2003, Polemical politics and the practice of community-organising in Cape Town, unpublished research report, $28 \mathrm{p}$.

OSTROM Elinor, 1992, Crafting Institutions for Self-governing Irrigation Systems, San francisco (CA), Institute for Contemporary Studies.

OSTROM Elinor, 1996, «Crossing the Great Divide : Coproduction, Synergy, and Development », in World Development, vol. 24, nº 6, pp. 1073-1087.

PAGE Ben, 2002, Has widening participation in decision-making influenced water policy in the UK?, Oxford, School of Geography and the Environment (University of Oxford), 27 p. (Working Papers in Economic Geography), www.geog.ox.ac.uk/research/wpapers/economic/wpg02-08.html

PAGE Ben, 2003, «Communities as the agents of commodification : the Kumbo Water Authority in Northwest Cameroon », in Geoforum, 34, pp. 483-498.

PAMUK Ayse, 2000, «Informal Institutional Arrangements in Credit, Land Markets and Infrastructure Delivery in Trinidad », in International Journal of Urban and Regional Research, vol. 24.2, pp. 379-396.

PEYROUX Élisabeth, 2000, Politiques d'habitat et pratiques résidentielles à Windhoek (Namibie): recompositions sociales et spatiales des périphéries d'une ville post-apartheid, Nanterre, Université paris X-Nanterre, 429 p. (thèse de doctorat).

PIROTTE Gautier, PONCELET Marc, 2002, «L'éveil des sociétés civiles en Afrique subsaharienne à la lumière des organisations non gouvernementales œuvrant en milieu urbain. Deux études de cas : Cotonou (Bénin) et Lubumbashi (RDC) », in Autrepart, $n^{\circ}$ 23, pp. 73-88.

SCHOU Arild, 1999, «Responsive Local Government : Does Democracy Matter ? A Comparison of a Zimbabwean and a Tanzanian Council », in $9^{e}$ conférence générale de l'EADI, Paris 22-25 septembre 1999, Paris, GEMDEV, 27 p. (communication $\mathrm{n}^{\circ} 317$ ).

SUBRAMANIAN Ashok et al., 1997, User Organizations for Sustainable Water Services, Washington, The World Bank, 162 p. (World Bank Technical Paper, n 354).

TENDLER Judith, 1997, Good Government in the Tropics, Baltimore, The Johns Hopkins University Press, 221 p. (The Johns Hopkins studies in development).

TOSTENSEN Arne et al., 2001, Associational Life in African Cities : Popular Responses to the Urban Crisis, Stockholm, Nordiska Afrikainstitutet, printed by Elanders Gotab, 324 p.

TUNSTALL Rebecca, 2001, «Devolution and User Participation in Public Services : How They Work and What They Do ? », in Urban Studies, vol. 38, n 13, pp. 2495-2514. 
Urban Management, November 1997, vol. 28, $\mathrm{n}^{\circ}$ 11, pp. 36-38 (« Up close : a look at the Durban tank and semi-pressure system »).

WORLD BANK, 1993, Water Resources Management Policy Paper, Washington, The World Bank, 141 p. (A World Bank Policy Paper).

WORLD BANK, 1997, World Development Report 1997: The State in a Changing World, New York, Oxford University press. 